\title{
A Case of Successful Twin Pregnancy Placed in Separate Horns of Bicornuate, Unicollis Uterus
}

\author{
A. W. Kuczynski ${ }^{1}$, W. Szcześniak ${ }^{1}$, A. Szczygielski ${ }^{2}$ \\ ${ }^{1}$ Department of Obstetrics and Gynecology, Regional Hospital in Drezdenko; ${ }^{2}$ Institute \\ of Obstetrics and Gynecology, Pomeranian Medical Academy, Szczecin, Poland
}

\begin{abstract}
We described a case of pregnancy, delivery and peurperium of 28 years old multipara with twins pregnancy in bicornuate, unicollis uterus. Each fetus was placed in separate horn of uterus. Biological mature pregnancy was delivered by cesarean section. During the peurperium the involution disturbances of one of the uterus horns were observed. The case was discussed in context of other similar anomalies.
\end{abstract}

Keywords: Bicornuate (uterus), Cesarean section, Infertility, Pregnancy, Twins, Uterus (abnormalities)

\section{INTRODUCTION}

The developmental anomalies of uterus and vagina caused by congenital Müllerian anomalies are observed in rate of about $0,7-1 \%$. In the lighter cases it becomes as uterus arcuatus with complete or partial septum of body, but in the severe cases it becomes uterus didelphys bicollis with partial vaginal septu. Bicornuate, unicollis uterus is a medium stage of mentioned abnormality. The pregnancy in separated horns of uterus usually ends in $1 / 2$ of cases with miscarriage or premature labor because of structural weakness of muscular coat and insufficiency of perfusion $[6,15]$.

In accessible literature we could not find any rapport of successful delivery of twins placed in separate horns of bicornuate uterus.

\section{CASE REPORT}

E.B. - a 28 years old multipara (file number 4380/98) was admitted for routine control examination as an outpatient at $6^{\text {th }}$ week after the fast menstruation period (11.04.1998). 


\section{A.W. Kuczynski et al.}

\section{Anamnesis}

Menarche when she was 14 , regular 26-day cycles, 5 -day painless poor menstruation. No abortions. She delivered once in 1997 by cesarean section because of pelvic longitudinal lie of fetus. Newborn weighed $2850 \mathrm{~g}$. During the operation the abnormality of uterus as bicornuate, unicollis uterus was stated, and the fetus was placed in the left horn of uterus. Postoperative stage without complications. The child is alive and until now its developing is normal.

At the admission a pulpiness and an enlargement of both horns of uterus were observed. Ultrasonography examination indicated initial suspicion of twin pregnancy with each fetus placed in different horn of uterus. General condition and laboratory tests were normal.

Because the pregnant refused to stay in hospital she was treated as an outpatient. The course of I and II trimester of pregnancy was normal.

On 24.11.1998 at 33 weeks of pregnancy she was admitted to hospital because of premature labor symptoms (uterine contractions, shortness and dilatation of the cervix). The labor activity were stopped by applying betamimetic drug (partusisten) intravenously at first, and then orally. In addition Isoptin and spasmolitics were applied. To speed up the development of fetal respiratory system corticosteroids were given.

The ultrasonography examination performed on 25.11.1998 resulted in:

\begin{tabular}{lll}
\hline & Fetus 1 & Fetus 2 \\
\hline BPD & $78 \mathrm{~mm}$ & $80 \mathrm{~mm}$ \\
FL & $59 \mathrm{~mm}$ & $62 \mathrm{~mm}$ \\
HC & $281 \mathrm{~mm}$ & $272 \mathrm{~mm}$ \\
AC & $271 \mathrm{~mm}$ & $260 \mathrm{~mm}$ \\
TBD & $84 \mathrm{~mm}$ & $83 \mathrm{~mm}$ \\
EFW & $1921 \mathrm{~g}$ & $1981 \mathrm{~g}$ \\
PL & II grade (Grannum) a front wall & II grade (Grannum) a fundus of uterus \\
AFI & $126 \mathrm{~mm}$ & $118 \mathrm{~mm}$ \\
\hline
\end{tabular}

The Manning test of both fetuses was normal.

After few days of treatment the symptoms of premature delivery disappeared, but the pregnant was still staying in the hospital.

At 36 weeks of pregnancy the Doppler US examination was performed in the Institute of Obstetrics and Gynecology MA in Szczecin. Normal indicators S/D, PI, RI in umbilical vessels and in middle cerebral artery of both fetuses were stated.

On 31.12.1998 at 39 weeks of pregnancy the cesarean section was performed because of premature rupture of membranes. Two male fetuses were delivered. First: weighed $2600 \mathrm{~g}, 49 \mathrm{~cm}$ long, Apgar score 9/9/10. The second: weighted $2400 \mathrm{~g}, 49 \mathrm{~cm}$ long, Apgar score 9/9/10. Both fetuses were in vertex-longitudinal lie. During the initial physical examination any developmental abnormalities were not observed, and the early adaptation period underwent without serious problems. 
During the postoperative stage the subinvolution of left horn of uterus was observed, and successfully treated pharmacologically.

The mother was discharged from hospital with her healthy children at $14^{\text {th }}$ day after the operation.

\section{DISCUSSION}

In whole pregnant population, women with developmental abnormalities account for $0,1-3,3 \%$.

$3,7 \%$ of them represent cases of bicornuate uterus $[3,12]$.

The uterine malformations are a significant factor of obstetrical risk, reason of primary sterility or infertility. Raga [15] says that infertile patients $(6,3 \%)$ and sterile women $(2,4 \%)$ have Müllerian abnormalities. The pregnancy as usual is result of assisted reproduction procedures $[2,3,7,12]$.

Frequently occurred complications are: imminent abortions $[5,10]$ or premature labor with secondary cervical insufficiency which require cerclage, not devoid risk [5, $12,15]$. There is another important risk, described by Chang [4], the rupture of horn containing ovum in the second trimester of pregnancy.

During the delivery, the weakness or dissociation of uterine activity and total or partial atony after the third stage of labor can occur.

A method of delivery prevalently used is the cesarean section $[1,4,5,13,14]$.

Raga [15] stated that the live birth rate of newborns delivered by women with bicornuate uterus is only $62,5 \%$, and a percentage of fetuses and infants with developmental abnormalities is significantly higher than in population of healthy pregnant women.

Nasal hypoplasia, omphalocele, limb deficiencies, teratomas and acardia-anencephaly also frequently occur [11].

Thanks to the development of ultrasonography techniques, enriched by hysteroscopy the recognition of developmental abnormalities of uterus became possible before and during pregnancy and some operations are now possible without laparotomy $[5,8$, $10,14]$.

There are only few descriptions of twin pregnancy in uterus with Müllerian abnormalities which occur the most frequently after assisted reproductions procedures. Barnet and al. [2] describe, as they stated, the only case of twin pregnancy in separate horn of uterus as a result of IVF and ET. Nahra-Lynch and al. [12] describe a similar case in one horn of uterus after the induction of ovulation and artificial insemination.

As we mentioned above there is no description of similar case in the literature, so we recommend this report to our readers, and we posit that the spontaneous biological carrying to term of twin pregnancy placed in separate horns of bicornuate uterus is possible. 


\section{A.W. Kuczynski et al.}

\section{REFERENCES}

1. Arredondo-Soberon $\mathrm{F}$ et al. (1997): Uterine arteriovenous malformation in a patient with recurrent pregnancy loss and a bicornuate uterus. A case report. J Reprod Med 42 (4): 239-43.

2. Barmet LJ et al. (1996): Twin gestation occupying separate horns of a bicornuate uterus after in-vitro fertilization and embryo-transfer. Hum Reprod 11 (10): 2316-8.

3. Check JH et al. (1992): Successful delivery of twins in a woman with a unicornuate uterus. Gynecol Obstet Invest 34 (2): 124-5.

4. Chang WF et al. (1994): Ultrasound diagnosis of rudimentary uterine horn pregnancy in fourteen weeks of gestation: a case report. Asia Oceania J Obstet Gynaecol 20 (3): 279-82.

5. Colacurci $\mathrm{N}$ et al. (1996): Reproductive outcome after hysteroscopic metroplasty. Eur J Obstet Gynecol Reprod Biol 66 (2): 147-50.

6. Gerbie MV (1980): General gynecological problems in pregnancy. Obstetrical practice. Sylvio Aldajem (Ed, the CV Mosby Company, St Luis - Toronto-London) 715-725.

7. Ginsberg NA et al (1997): Management of a triplet gestation complicated by uterus didelphys. Fetal Diagn Ther 12 (1): 59-60.

8. Heinonen PK (1997): Reproductive performance of woman with uterine anomalies after abdominal or hysteroscopic metroplasty or no surgical treatment. J Am Assoc Gynecol Laparosc 4 (3): 311-7.

9. Homsi RI et al. (1994): Uterus didelphys and unilateral lower limb amelia in a discordant monozygotic twin. A case report. J Reprod Med 39 (6): 481-4.

10. Maneschi F et al. (1995): Hysteroscopically detected asymptomatic Müllerian anomalies. Prevalence and reproductive implications. J Reprod Med 40 (10): 684-8.

11. Martinez-Frias et al. (1998): Congenital anomalies in the offspring of mothers with a bicornuate uterus. Pediatrics 101 (4): E10.

12. Nahra-Lynch $M$ et al. (1997): Multiple gestation in a unicornuate uterus. A case report. J Reprod Med. 42 (7): 451-4.

13. Nahum GG (1997): Rudimentary uterine horn pregnancy. A case report on surviving twins delivered eight days apart. J Reprod Med 42 (8): 525-32.

14. Pelosi MA (1996): Laparoscopic-assisted transvaginal metroplasty for the treatment of bicornuate uterus: A case study. Fertil Steril 65 (4): 886-90.

15. Raga F et al. (1997): Reproductive impact of congenital Müllerian anomalies. Human Reprod 12 (10): 2277-81.

Correspondence: A.W. Kuczynski, Department of Obstetrics and Gynecology, Regional Hospital in Drezdenko, U1. Marszałkowska 3, 66-530 Drezdenko, Poland. 